\title{
Aperçu
}

\section{Surveillance aux services d'urgence des blessures à la tête et de l'ensemble des blessures provoquées par le baseball, le football, le soccer ou le hockey sur glace chez les enfants et les jeunes de 5 à 18 ans, 2004 à 2014}

\author{
S. McFaull, M. Sc.; J. Subaskaran, M.H.P.; B. Branchard; W. Thompson, M. Sc.
}

Diffuser cet article sur Twitter

Les sports d'équipe sont populaires auprès des jeunes Canadiens. La figure 1 présente, pour 11 ans (2004 à 2014), le nombre et la proportion (sur 100 000) de blessures ainsi que de blessures à la tête subies par les enfants et les jeunes de 5 à 18 ans ayant pratiqué l'un des quatre sports d'équipe suivants : baseball, football, soccer et hockey sur glace. Les données recueillies dans le Système canadien hospitalier d'information et de recherche en prévention des traumatismes (SCHIRPT) ${ }^{1}$, un système de surveillance sur les blessures et les intoxications géré par l'Agence de la santé publique du Canada, ont été utilisées pour créer la figure 1 (tableaux disponibles sur demande). Le SCHIRPT est actuellement utilisé dans 11 hôpitaux pour enfants et 6 hôpitaux généraux au Canada et comporte un système de saisie des données en ligne mis à jour quotidiennement par l'intégration des nouveaux cas et de l'information pertinente. On n'y saisit que les blessures des patients se présentant aux urgences des hôpitaux participants, ce qui fait qu'il ne s'agit pas de données exhaustives pour l'ensemble du Canada. Différentes études montrent néanmoins que les modèles qui en résultent sont représentatifs de la situation canadienne dans certains contextes ${ }^{2,3}$. Tous les cas jugés non pertinents ou contenant des erreurs ont été exclus de l'analyse.

Le pourcentage annuel moyen de variation de toutes les blessures signalées par l'entremise du SCHIRPT a été calculé (avec des intervalles de confiance [IC] à $95 \%$ ) pour chacun des sports, conformément à la méthodologie du National Cancer Institute ${ }^{4}$. Sur 11 ans, la proportion de toutes les blessures (blessures totales pour 100000 cas dans le SCHIRPT) attribuables au baseball est demeurée stable. Les blessures causées par le football sont demeurées globalement stables, mais leur proportion a grimpé à environ $7 \%$ (IC à $95 \%: 3,1$ à 11,0 ) par année de 2004 à 2008 puis a diminué de 2,2\% (IC à $95 \%:-3,9$ à -0,5) par année de 2008 à 2014. Les blessures attribuables au soccer sont elles aussi demeurées globalement stables, malgré une hausse de 1,9\% (IC à $95 \%$ : 0,6 à 3,2) de 2007 à 2014. Les blessures attribuables au hockey sur glace sont demeurées relativement stables sur 11 ans, mais une tendance à la hausse de $7,7 \%$ (IC à $95 \%$ : 5,9 à 9,6) par année a été observée pour 2006 à 2011.

De façon générale, c'est le baseball qui engendre la plus grande proportion de blessures à la tête signalées (par rapport à l'ensemble des blessures), avec un pourcentage de $35,0 \%$ (1854/5300), suivi du hockey sur glace avec 27,2 \% (11 425/42 029), du football avec 16,3\% (3635/22 264) et du soccer avec 15,9 \% (7326/46 102). À l'exception du baseball, dont les chiffres sont demeurés relativement stables, la proportion de blessures à la tête causées par le football, le soccer et le hockey sur glace a augmenté entre 2004 et 2014 dans une fourchette allant de $42 \%$ à $47 \%$.

L'étude comporte certaines limites : l'augmentation des blessures pourrait s'expliquer en partie ou en totalité par la participation accrue à des activités sportives ou encore par l'augmentation du nombre de cas se présentant aux urgences. On ne peut pas nécessairement conclure à une augmentation du danger ou des risques liés au sport. De même, la proportion accrue, au fil des ans, des blessures à la tête pourrait être attribuable à l'augmentation des proportions déclarées, à l'augmentation des déclarations par l'entremise du SCHIRPT comme à la diminution du nombre de blessures ailleurs qu'à la tête. 
FIGURE 1


enfants et jeunes de 5 à 18 ans, 2004 à $2014^{c}$

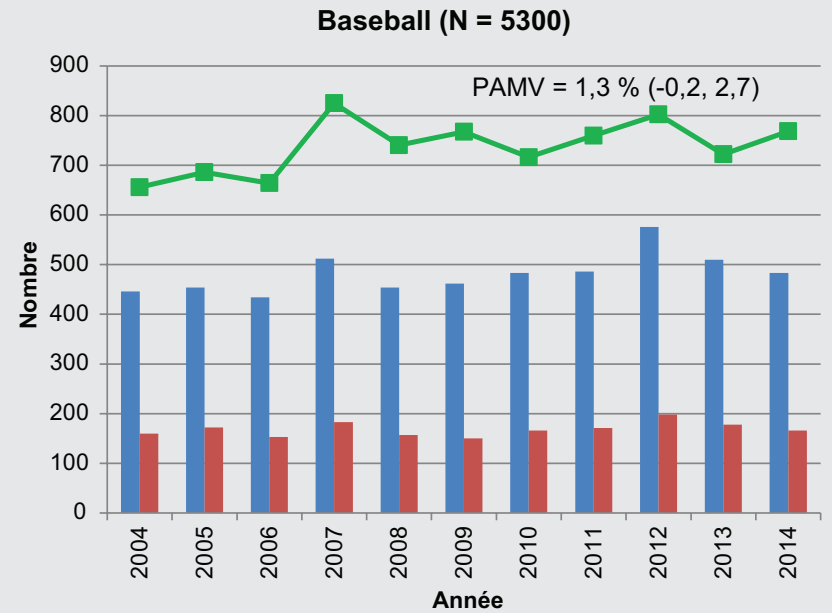

Toutes les blessures Blessures à la tête - Nombre pour 100000

Soccer $(N=46$ 102)

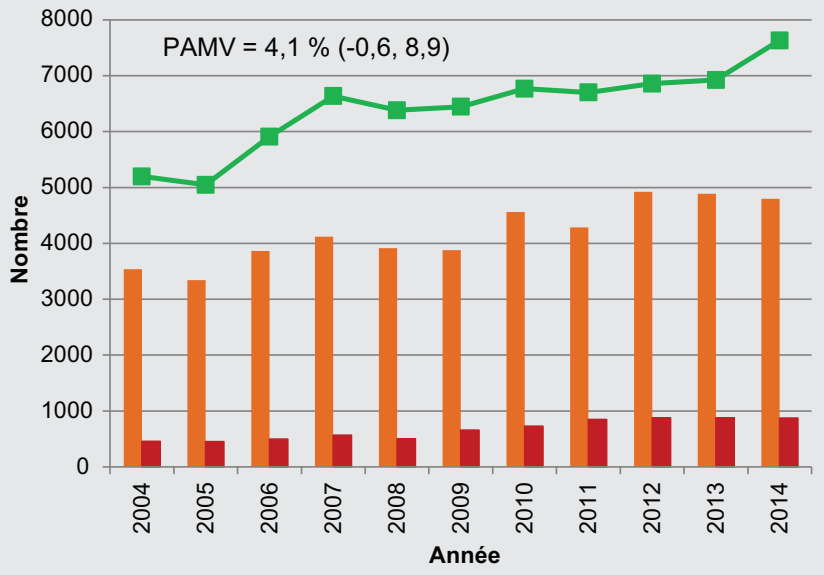

Toutes les blessures $\quad$ Blessures à la tête $\rightarrow$ Nombre pour 100000
Football $(\mathrm{N}=22$ 264)

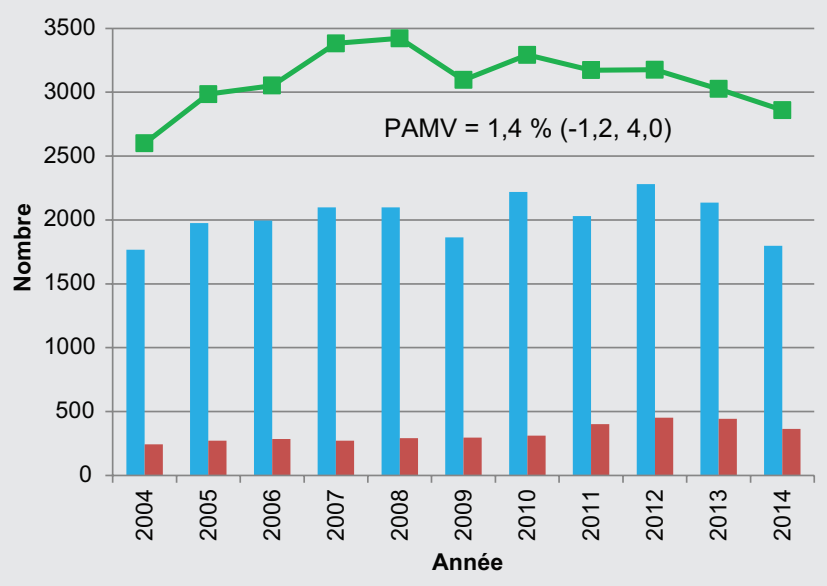

Toutes les blessures Blessures à la tête - Nombre pour 100000

Hockey sur glace ( $N=42$ 029)

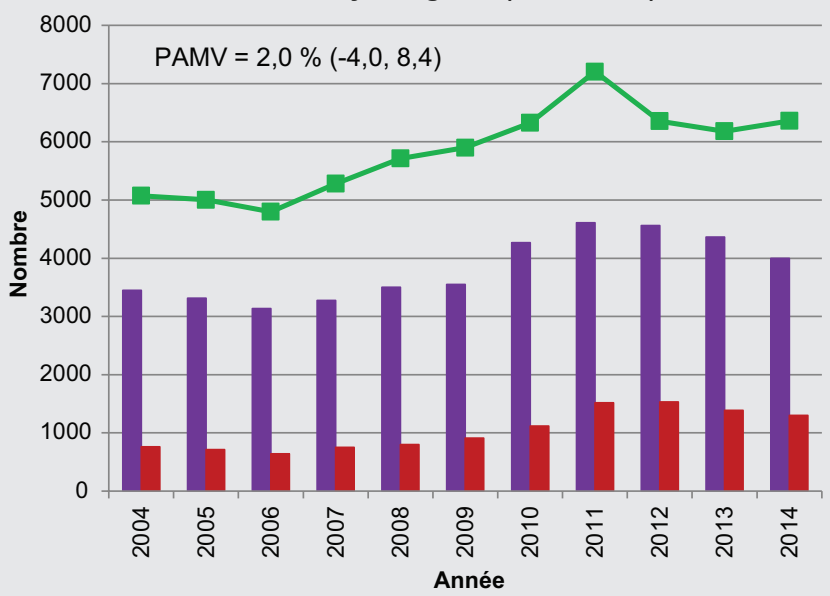

Toutes les blessures Blessures à la tête - Nombre pour 100000

Abréviations : PAMV, pourcentage annuel moyen de variation; SCHIRPT, Système canadien hospitalier d'information et de recherche en prévention des traumatismes. Remarque : Le PAMV de toutes les blessures signalées a été calculé avec des intervalles de confiance à $95 \%$.

${ }^{a}$ Comprend : fractures du crâne ou du visage, lacérations du crâne ou du visage, blessures aux dents et traumatismes cérébraux (légers traumatismes crâniens fermés, commotions et blessures intracrâniennes).

${ }^{\text {b }}$ Nombre de blessures pour 100000 cas de tous les types de blessures chez les 5 à 18 ans pour l'année en cause dans le SCHIRPT.

${ }^{\text {c }} \mathrm{Au} 15$ juin 2015. Les chiffres pour 2012 à 2014 sont des estimations proportionnelles étant donné que de l'information continue d'être saisie dans le SCHIRPT.

\section{Références}

1. Mackenzie SG, Pless IB. CHIRPP: Canada's principal injury surveillance program. Inj Prev. 1999;5:208-213.

2. Pickett W, Brison RJ, Mackenzie SG et collab. Youth injury data in the Canadian Hospitals Injury Reporting and Prevention Program: do they represent the Canadian experience? Inj Prev. 2000;6:9-15.
3. Kang J, Hagel B, Emery CA, Senger T, Meeuwisse W. Assessing the representativeness of Canadian Hospitals Injury Reporting and Prevention Program (CHIRPP) sport and recreational injury data in Calgary, Canada. International Journal of Injury Control and Safety Promotion. 2013;20(1): 19-26.
4. Average Annual Percent Change (AAPC) [Internet]. National Cancer Institute; [consultation le 3 septembre 2015]. Consultable en ligne à la page http://surveillance.cancer.gov/ joinpoint/webhelp/Executing_the_Joinpoint_ Parameters/Statistical_Notes/Statistics_Relat ed_to_the_k-joinpoint_Model/Average_Annu al_Percent_Change.htm 